\title{
Interactive comment on "Optimising Seasonal Streamflow Forecast Lead Time for Operational Decision Making in Australia" by A. Schepen et al.
}

\section{Anonymous Referee \#1}

Received and published: 10 June 2016

General Comments This paper proposes a new forecast technique for water supply forecasting in Australia designed to allow for more frequent updates as well as to improve forecast accuracy and to make the forecasts more timely. Currently operational forecasts are released 7 days into a forecast target period. This proposed technique would include the entire forecast period in the future. Overall, the paper was logically organized and easy to follow. The verification metrics used were applicable to the problem and applied in a logical manner. The results supported the conclusions in the paper well. I am curious why the authors did not investigate incorporation of weather prediction into their algorithm. This would seem to be a major area ripe for improvement as the forecast skill at the weather timescale (0-2 weeks into the future) is much larger than at climate time scales and is ever increasing as computer power continues to grow. It was striking that there was no discussion on how forecast utilization. I rec- 
ommend including information on how the current forecasts are utilized and how the new forecasts might improve application. Specific comments [page 3; lines 9-11] How is the undesirability of forecasts beyond 1 one month consistent with the premise of this paper of improving 3 month forecasts? [page 3; line 13] What is $\mathrm{N}$ equal to? [page 4 ; line 23] is daily updated subsurface temperatures really necessary for this method? Does it change that much from one day to the next?

Interactive comment on Hydrol. Earth Syst. Sci. Discuss., doi:10.5194/hess-2016-199, 2016. 